\title{
LUDWIG BINSWANGER ET LA PHÉNOMÉNOLOGIE
}

\author{
François De Gandt \\ Université Charles de Gaulle Lille III \\ UMR Savoirs Textes Langages
}

Ludwig Binswanger, psychiatre suisse de langue allemande (1881-1966), a laissé une œuvre qui est une source d'inspiration exceptionnelle. Un trait essentiel est l'usage qu'il fait de la phénoménologie dans la description et la théorisation des cas cliniques. Mais qu'appelle-t-il phénoménologie ? Tout au long de son développement intellectuel et dans les diverses phases de son travail, on voit se transformer le statut qu'il donne à la phénoménologie, l'usage qu'il en fait, la sorte de phénoménologie à laquelle il se réfère, Depuis l'article introductif de 1922 "De la phénoménologie", puis dans "La fuite des idées" de 1933 par exemple, enfin dans "Délire" de 1965, à la veille de sa mort, le recours à la phénoménologie est très différent.

Le présent exposé sera plutôt une introduction, pour aider à entrer dans l'oeuvre de Binswanger, une oeuvre puissante, passionnante, mais mal connue et difficile d'accès. Elle est particulièrement féconde par le croisement qu'elle opère entre plusieurs disciplines, mais cela en fait aussi la difficulté.

\section{Le métier de psychiatre}

Qui est Binswanger? Voici une anecdote qui dira beaucoup: dans son avant dernier livre paru en 1960, Manie et mélancolie, Binswanger commence par le cas d'une certaine Cécile Münch, si triste et si marquée par la douleur, et il ajoute:

Durant toute ma vie j'ai revu la "figure gémissante" 1 et le regard profondément mélancolique de cette femme si nettement devant moi, que j’étais extrê-

1 En allemand "Jammergestalt", qu'on pourrait traduire aussi par "figure de la détresse" ou "incarnation de la plainte". 
mement surpris de constater, au cours de mes recherches, qu'au moment de son séjour je n'avais que dix ans. Que j'aie pu être si profondément impressionné par cette figure, par ce destin, vient de ce qu'encore enfants nous étions souvent avec nos malades et nous apprenions beaucoup sur leur histoire à travers les discussions des adultes. ${ }^{2}$

Cette impression qui lui revient si fortement en 1960 - il a 79 ans -, c'est le visage douloureux d'une pauvre femme entrée "chez nous", comme il dit, en 1891, alors qu'enfant il vivait au milieu des malades de la clinique paternelle.

Binswanger est d'abord un psychiatre de métier, ancré dans une pratique clinique quotidienne, ancré aussi dans une institution et une tradition, le troisième d'une dynastie de psychiatres, attachés de père en fils sur quatre générations à la vie d'un établissement de séjour et de soins, la clinique Bellevue de Kreuzlingen, fondée par son grand père Ludwig en 1857 et dont lui-même confiera la direction à son fils Wolfgang en 1960. La clinique disparaîtra en 1984 après environ 130 ans d'existence.

Ludwig devient directeur à la mort de son père en 1911, il n'a que 30 ans. Après un stage chez Kraepelin à Heidelberg, il a passé sa thèse en 1907 sous la direction de C.G. Jung et reste très proche de Bleuler, le patron du Burghölzli de Zürich, mais il refusera toujours une chaire universitaire. Il a été en fonction chez son oncle Otto, psychiatre lui aussi à Iena, célèbre notamment pour avoir tenté de soigner Nietzsche en 1889. C'est chez son oncle Otto qu'il a connu sa femme Herta, qui était infirmière.

Les méthodes de soins de la clinique Bellevue étaient très originales et inventives. On y donnait une attention particulière à chaque malade, on essayait toutes sortes de techniques et de pratiques, comme les thérapies par la parole, l'hydrothérapie, le sport, l'expression artistique, on organisait des excursions et des fêtes, on tâchait de créer autour des malades une ambiance rassurante et riche, pour "repossibiliser" leur existence arrêtée - aujourd'hui on dirait qu'il s'agissait d'un "lieu de vie". 3

Cette activité professionnelle a été pour Ludwig intimement jointe à un souci théorique constant: fonder la psychiatrie, avec cette question simple et forte: qu'est-ce qu'un homme? Cette interrogation se nourrissait chez Binswanger d'une culture humaniste vivante, très solide et vaste, incluant la philosophie, la poésie, la littérature ancienne, le théâtre. Il recevait à Bellevue des amis médecins, savants et philosophes, comme Minkowski, Staiger, Häberlin, Szilasi, Kuhn. Des penseurs célèbres comme Buber, Heidegger et Husserl sont venus à Bellevue eux aussi. Ses liens personnels avec

2 Manie et mélancolie, trad française par Azorin, Totoyan et Tatossian, PUF 1987, p. 30.

3 On pourra se faire une idée de la clinique quotidienne à Bellevue en lisant le recueil de lettres et de documents consacré au séjour d'Aby Warburg à Bellevue (1921-1924): Ludwig Binswanger et Aby Warburg, La guérison infinie, Paris, Payot 2007. 
Freud, depuis une mémorable visite à Vienne en compagnie de Jung, mériteraient un développement particulier.

Cet effort de cinquante années pour fonder la psychiatrie a donné naissance à une oeuvre écrite très vaste, qui compte 172 titres ${ }^{4}$ si l'on inclut les livres, articles, rapports, essais. Les textes sont de nature et de style très différents, depuis des rapports de 40 pages présentés devant des congrès de psychiatrie, jusqu'à un très ambitieux traité d'anthropologie de 700 pages, publié en 1942, Grundformen und Erkenntnis menschlichen Daseins. Les ruptures de style ne facilitent pas toujours la lecture, puisqu'on passe parfois abruptement, dans le cours du même ouvrage, d'une vignette clinique à une envolée philosophique ou à un compte-rendu de travaux récents.

Du début à la fin il a considéré la phénoménologie comme le soubassement scientifique indispensable à la psychiatrie. Ainsi déclare-t-il dans l'un de ses derniers textes, Mélancolie et manie (1960), que "la science husserlienne réalise pour la psychiatrie ce que la biologie réalise pour la médecine somatique". 5

\section{Trois périodes}

Il est utile, pour notre propos au moins, de distinguer dans l'œuvre de Binswanger trois périodes ou trois phases, selon ce qu'a représenté pour lui la phénoménologie:

A) Vers 1920 c'est la découverte de la phénoménologie à travers l'oeuvre de Husserl, et la formulation d'un programme. Le "rapport” prononcé en 1922 De la phénoménologie marque vigoureusement cette étape.

B) Puis c'est l'éblouissement du Sein und Zeit de Heidegger (1927), qui ouvre à Binswanger une voie riche et nouvelle, exploitée surtout dans les études de La fuite des idées(1933).

C) Enfin s'effectue une sorte de retour ou de reprise, dans les deux derniers livres assez courts, Mélancolie et manie (1960) et Délire (1965). Des lecteurs y ont vu un "retour à Husserl", mais Binswanger a récusé la formule, et tenté de montrer comment il faut distinguer et articuler les points de vue "daseinsanalytique" et "phénoménologique".

4 Voir la liste donnée dans Ludwig Binswanger, Ausgewählte Werke, Band 3, Vorträge und Aufsätze, Asanger Verlag 1994, pp. 359-373. Cette liste n'inclut pas les traductions.

${ }^{5}$ Manie et mélancolie, trad. française par Azorin, Totoyan et Tatossian, PUF 1987, p. 135. 
Si l'on veut se faire d'abord une idée très tranchée et brutale, toute provisoire et simpliste, on peut décrire ces trois phases par des formules: A) l'intuition; B) le Dasein et les styles d'être au monde; C) les ruptures dans l'enchaînement et les défauts de constitution. La phénoménologie apparaît dans ces trois phases sous un jour différent: une méthode d'intuition en 1922, la description de styles de présence en 1933, la description de la structure de l'expérience en 1960.

Ces distinctions et ces formules sont utiles pour aider à s'orienter, mais il faut aussi ressaisir l'unité d'un mouvement. Déjà on peut affiner ce panorama en insérant une sorte de phase intermédiaire entre 1933 et 1950, au cours de laquelle Binswanger prend une distance critique vis à vis de Heidegger, prépare son anthropologie exposée dans les Grundformen de 1942 et rédige cinq études de cas (de 1947 à 1952: Ellen West, Ilse, Jürg Zünd, Lola Voß, Suzanne Urban). C'est aussi le temps de l'étude sur Ibsen (1950) et des Trois formes manquées du Dasein (1956).

Le tableau ci-dessous visualise ce découpage et rassemble quelques titres plus marquants avec leur date et éventuellement la mention de leur traduction en français:

1907 visite chez Freud avec Jung;

1911 dir. de la Clinique Bellevue (cure Warburg 1921-4)

Première période

la phénoménologie comme méthode d'intuition

1922 Ueber Phänomenologie (trad. De la phénoménologie, in Intr. à l'analyse existentielle)

DEUXIĖME PÉRIODE

la description d'un style d'être au monde

1930 Traum und Existenz (trad. Rêve et existence 1954, repris in Intr. à l'analyse existentielle)

1933 Ueber Ideenflucht (tr. Sur la fuite des idées, 2000)

1933 Das Raumproblem in der Psychopathologie (trad. Le problème de l'espace, 1998)

Période intermédiaire

anthropologie, critique de Heidegger et analyses de cas

1942 Grundformen und Erkenntnis menschlichen Daseins (non trad.)

1944 Der Fall Ellen West; 1945 Ilse; 1946-7 Jürg Zünd; 1949 Lola Voß

1950 Hendrik Ibsen und das Problem der Selbstrealisation in der Kunst (trad. H. Ibsen, 1996) 
1952 Der Fall Suzanne Urban (trad. Le cas Suzanne Urban 1957)

1956 Drei Formen mißglückten Daseins: Verstiegenheit, Verschrobenheit, Manieriertheit (trad. Trois formes manquées de la présence humaine, 2002)

DERNIÈRE PÉRIODE

constitution de l'expérience délirante (retour à Husserl ?)

1960 Melancholie und Manie (tr. Mélancolie et manie 1987)

1965 Wahn (tr. Délire 1993)

\section{À la recherche d'une forme de connaissance}

L'article de 1922, De la phénoménologie, ${ }^{6}$ est un programme, et aussi le témoignage d'une sorte de choc. Dans ce rapport de synthèse adressé à un Congrès de psychiatres, Binswanger tente de faire 'connaître une méthode ou plutôt une orientation nouvelle en psychiatrie. Le mot orientation convient assez bien, parce que la question est de rendre possible d'autres procédures et d'autres voies d'accès que celles de la psychopathologie d'inspiration objectivante.

D'abord Binswanger énonce quelques précautions apparemment rhétoriques. Un rapport est-il le bon moyen pour permettre une entrée dans la phénoménologie ? Peut-on prendre conscience d'une possibilité nouvelle d'orientation simplement en écoutant un rapport à un Congrès, qui d'habitude se contente de rassembler des résultats utiles, de proposer un bilan de travaux antérieurs et de donner des aperçus ("Uebersichtreferate") ? Il faudra ici viser un autre but: rendre sensible l'objet, faire apparaître quelque chose à l'intuition, installer une réalité devant l'œil de l'esprit ("vor das geistige Auge zu bringen"). Ce ne sera pas complet au sens de la complétude d'un rapport, mais peut-être au sens de la complétude d'une "vue" ou d'une "intuition" ("Vollständigkeit der Anschaulichkeit"). ${ }^{7}$ Nous sommes invités à un effort pour voir.

Comparons en premier lieu la science de la nature et la phénoménologie. Les psychiatres sont des hommes de science, mais que signifie cette prétention? La psychiatrie peut-elle être une branche des sciences de la nature? Peut-elle admettre les procédés méthodiques des sciences de la nature? Dès le début de son rapport, Binswanger situe comme un repoussoir la méthode de la science de la nature (la pratique des "Naturforscher"). Cette science

${ }^{6}$ Le texte allemand est reproduit dans le volume III des œuvres choisies de Binswanger: Ludwig Binswanger, Ausgewählte Werke, Band 3, Vorträge und Aufsätze, Asanger Verlag 1994, pp. 34-69. Voir la traduction française, dans Ludwig Binswanger, Introduction à l'analyse existentielle, Éd. de Minuit, Paris 1971, pp. 79-117. Mais cette traduction est souvent très imprécise, pour un texte qui mérite d'être lu de très près.

7 Texte allemand, op. cit., p. 35. 
s'occupe de tout le réel-dans lequel se trouve aussi le savant lui-même comme objet oeuvrant et souffrant-, elle est aux prises avec le double monde des faits physiques et psychiques ("diese Welt, eingeteilt in eine Welt körperlicher und seelischer Tatsachen") qui font une seule nature, mais elle s'est donné une méthode qui suppose des limitations volontaires: elle s'en tient à l'observable, elle décompose ses objets. En des termes plus précis:

1) elle n'admet que ce qui est vérifiable par des constatations objectives et sensibles, elle se restreint donc volontairement et méthodiquement à ce qui est accessible par la perception externe ou interne ("allein vermittels der sinnlichen (äußeren oder inneren) Wahrnehmung"). Pour elle il n'y a pas d'autres voies d'accès recevable. La science de la nature ne connaît pas d'autre connaissance "directe ou primaire".

2) elle décompose ("zerlegt") en éléments tout objet ou tout processus ("Ding oder Vorgang"), elle les pulvérise en leurs "propriétés, éléments ou fonctions".

\section{La vision directe de l'artiste}

Faut-il s'en tenir à cette forme de constatation objective et analytique ? Il y a peut-être une autre voie, une autre sorte de connaissance, cette fois "immédiate et directe", la voie d'une "saisie spirituelle plus totale et plus originaire" ("ein anderes, ursrprünglicheres und totaleres geistiges Erfassen" "). Il y a en effet des hommes qui savent qu'autre chose est possible, il y a des observateurs et créateurs qui s'y prennent autrement: ce sont les artistes.

"Contempler, regarder et regarder encore", voilà ce que fait l'artiste ("Betrachten, Schauen und nochmals Schauen"). Cet effort de saisie directe aboutit parfois, miraculeusement, à une "entrée" dans l'objet ("Hineinsetztwerden", qu'on pourrait traduire par "se retrouver installé à l'intérieur de l'objet"). Binswanger cite cette phrase surprenante de Flaubert: "À force de regarder un caillou, un animal, un tableau, je me suis senti y entrer".

Et il ne suffit pas d'avoir vu, il faut encore traduire la vision, en une œuvre que d'autres comprendront et qui enrichira leur regard, leur apprendra à voir autrement à leur tour. Lorsqu'il a vu, le créateur restitue ou transmet sa vision, qui ne ressemble pas à ce qu'on voit d'habitude. Franz Marc, par exemple, peint des chevaux bleus. Il rend ainsi manifeste ("darstellt") une propriété des chevaux qu'on ne trouve pas dans la nature, parce que cette propriété ne peut se percevoir sur le mode sensible usuel. Le peintre semble contredire la nature, et pourtant il a "vu" quelque chose. Il a vu ce qu'on ne voit pas d'habitude, en regardant les chevaux l'un après l'autre, il a vu l'essence du cheval ("das 
Wesen des Pferdartigen"). De même pour un chevreuil, il a vu quelque chose du chevreuil, non pas ce qu'on voit par les sens, mais une essence de chevreuil ("das Rehartige"), ce qui fait que par exemple une jeune fille a quelque chose de commun avec le chevreuil.

Quand Van Gogh peint un arbre fouetté par le vent, il exprime un drame, et quand il peint un champ de blé, il rend visible quelque chose que le champ de blé a en commun avec un enfant qui dort.

Ce n'est pas un acte de voir au sens de la perception sensible, et pourtant il y une saisie immédiate, qui n'est pas moins convaincante et qui même possède plus de certitude immédiate que la perception sensible. Dès lors que l'on en possède la capacité spirituelle, on peut accéder à cette vue, effectuer l'acte de voir ce qu'il y a à voir. ${ }^{9}$

La peinture n'est pas la seule voie. Quand Dostoievski, dans le Double, décrit une psychose commençante, il ne respecte pas ce qu'on trouve d'habitude dans l'expérience clinique. Pourtant il a vu quelque chose et l'a amené à l'expression, il a traduit ce que les malades eux-mêmes attestent, et qui est absent des manuels psychiatriques: le phénomène de la désinstallation du Moi, du déplacement ou du dérangement ("Verrückung des Ich"). Le moi est saisi par une autre puissance, une loi nouvelle, inconnue et effrayante.

Les musiciens aussi savent voir et faire voir - ou donner à entendre -, comme Debussy exprimant l'essence d'une pagode ou d'une cathédrale engloutie.

Deux remarques ici: l'objet appréhendé, vu par une vue spirituelle, ce n'est pas l'individu - ce cheval particulier, ou cette terreur de devenir fou, ou cette pagode -, c'est une essence, une généralité; l'artiste va droit à un caractère général, il trouve et exprime ce qui dépasse l'individuel. ${ }^{10} \mathrm{Et}$ l'un des critères auxquels on peut reconnaître cet essentiel, c'est qu'il est transposable par métaphore: on sait que l'artiste a atteint l'essence du champ de blé ou du chevreuil, dès lors qu'il a exprimé du même coup ce qui est commun entre un champ de blé et un enfant qui dort, ou entre un chevreuil et une jeune fille. Comme si la métaphore était le milieu de subsistance normal des essences.

Binswanger demande: pourrait-on expliquer tout cela par un processus d'association, à la manière de la psychologie usuelle? Mais un rappel associatif suppose une première fois: si quelqu'un est amené par association à unir l'impression du champ de blé et celle de l'enfant qui dort, cela suppose qu'il ait déjà, au moins une fois, perçu le "phénomène" unitaire, qui s'appliquera

9 Texte allemand, op. cit., p. 37.

10 Par opposition avec l'intuition bergsonienne par exemple, qui est supposée atteindre ce que l'individu a d'unique. 
aux deux situations et que l'on pourrait appeler "le pur, le doux, l'émouvant"." L'association doit être fondée, rendue possible par une saisie préalable.

\section{L'intuition en phénoménologie}

La pratique des artistes enseigne qu'il est possible de voir autrement. Mais la phénoménologie n'est pas tout à fait du même ordre que l'art. Le détour par l'effort des artistes permet au moins de réouvrir le regard, de convaincre qu'il existe d'autres formes de saisie, et que "notre connaissance intuitive ou immédiate va infiniment plus loin que la fonction et le domaine de la perception sensible". ${ }^{12}$

C'est la phénoménologie de Husserl qui peut prendre en charge, conceptuellement et systématiquement, cette ouverture plus large du regard, en justifiant l'existence ou la légitimité de formes d'intuition non perceptives.

A cet endroit Husserl apporte un principe décisif: il y a diverses sortes de domaines d'objets et dans chaque cas il doit exister un acte approprié d'intuition. ${ }^{13}$ A chaque domaine son acte de saisie. Ce ne sera pas toujours une perception sensible, mais ce sera toujours une saisie directe, un mode de connaissance simple et ultime, qui peut être considéré comme la sorte d'évidence propre à ce domaine d'objets.

L'exemple premier et fondamental pour Binswanger (comme pour Heidegger) est celui de l'intuition "catégoriale". Que veut dire ce terme ? "Catégorial", qui est assez proche de "grammatical" ou "structurel", s'oppose à "matériel", "concret", et la présentation de l'intuition catégoriale provient de la Sixième Recherche Logique de Husserl. La question est de savoir comment on "remplit", on donne un sens à une expression. Husserl a montré dans cette Recherche VI à quelles conditions peut se faire le remplissement d'une visée signifiante: il faut pouvoir remplir non seulement les termes signifiants concrets et matériels, mais aussi les termes "catégoriaux".

Précisons: le remplissement d'une expression grammaticalement complexe suppose que l'auditeur ou le lecteur amène à l'évidence non seulement les objets concrets et sensibles nommés dans la phrase mais aussi les liaisons ou relations abstraites qui font partie de la phrase. Pour donner un sens à la phrase, il faut pouvoir "remplir" la visée signifiante qui porte sur les "matériaux", mais aussi la visée qui porte sur les "formes catégoriales" (conjonction,

11 “Zuerst muß das Phänomen des 'Reinen, Sanften, Rührenden' erschaut sein, bevor die Assoziation 'schlafendes Kind' erfolgen kann; erst auf Grund dieses Phänomens wird ja die Assoziation 'gestiftet'.", ibid., p. 40.

12 Texte allemand, op. cit., p. 38.

13 "Es gibt weite, sehr weite Gegenstandsgebiete, die der sinnlichen Wahrnehmung unzugänglich sind, und von denen wir doch anschauliche Kenntnis bekommen und verarbeiten; ja, wir dürfen sagen: daß sich für jedes Gegenstandsgebiet ein entsprechender Akt anschaulicher Kenntnisnahme finden läßt.", p. 38. 
disjonction, copule marquée par le verbe être, négation, etc.). Quand j'entends la phrase "le chat dort et il est sur le tapis", je comprends les mots plus concrets "chat", "dort" et "tapis", je "remplis" ces visées signitives de chat, de sommeil et de tapis par des saisies plus ou moins évidentes - qui sont en dernier ressort des perceptions sensibles: je vois le chat et je vois le tapis -, j'ai un accès à ce que cela "veut dire". Mais il faut aussi que je donne un sens à "et" et à "est sur", il faut que je "remplisse" intuitivement ces termes signifiants, mais dans ce cas ce ne peut être au moyen d'une perception sensible - je ne peux pas "voir" le "et" comme on voit un objet concret et matériel dans l'espace. Il doit pourtant bien y avoir une certaine sorte de saisie directe, d'évidence ultime et irréductible du "ou" du "et", du "ne pas", du "est", du "parce que". Il faut bien une sorte d'accès direct à ces formes, un accès qui soit l'équivalent de cette "intuition" qui amène des objets sensibles à l'évidence. Comme cette nouvelle sorte d'intuition porte non sur la matière ou le matériau, mais sur la structure des énoncés, sur le formel, ou encore le grammatical, que Husserl appelle le "catégorial" des énoncés, il nomme cela "intuition catégoriale". ${ }^{14}$

Binswanger s'empare de cet outil victorieusement, et au prix de quelques ambiguïtés. Il réunit assez sommairement deux sortes d'intuition qui nécessiteraient une distinction soigneuse: le catégorial et l'éidétique (un terme formé à partir d'eidos, qui signifie essence). La discussion précise et rigoureuse sur le lien et la différence entre catégorial et éidétique nous entraînerait trop loin.

Remarquons que Heidegger a lui aussi - durant ces mêmes années choisi cette thèse de Husserl comme point d'appui archimédien pour ouvrir la possibilité nouvelle d'une intuition non sensible, la possibilité d'une appréhension de l'être. La sixième Recherche Logique, avec cette notion d'intuition catégoriale, était pour lui et ses élèves une percée décisive. ${ }^{15}$

Peu importent ici, dans le présent contexte, les subtilités de la phénoménologie husserlienne. Binswanger insiste sur l'effort de voir, sur l'exercice de délivrance des préjugés et des préconceptions, sur l'exigence d'un retour toujours renouvelé aux choses mêmes, à ce qui est visé derrière les mots, pour tenter de vivre de l'intérieur l'expérience dont on parle.

\section{Un exemple: la salle de paroles}

Pour illustrer l'exercice de saisie intuitive auquel il invite ses collègues psychiatres, Binswanger propose l'exemple d'une malade qui entend des voix.

14 Binswanger cite ici un long passage de la $6^{\mathrm{c}}$ Recherche Logique de Husserl, dont le passage décisif pourrait être celui-ci: "il faut qu'il y ait, pour les éléments catégoriaux de la signification, un acte qui rende le même service que la simple perception sensible le fait pour les composantes matérielles de la signification" (p. 39 note).

15 Voir par exemple Heidegger Prolegomena zur Geschichte des Zeitsbegriffs (cours de 1925) G.W., volume $20, \S 6$. 
On peut dresser un diagnostic, classer le malade dans une catégorie particulière de délirant ou d'anormal, chercher une explication de divers côtés, on peut aussi tenter de pénétrer plus profondément. Questionnée, la malade dira:

Nein, Stimmen höre ich nicht, aber nachts sind Sprechsäle eröffnet, die ich gerne würde dispensieren. ${ }^{16}$

ce qu'on pourrait traduire par: "Non, je n'entends pas des voix, mais la nuit des parloirs sont ouverts, je préfèrerais qu'ils soient mis en congé”.

La seule entreprise de traduire correctement suppose déjà un exercice de compréhension et de vision. Que représentent pour un malade ces "Sprechsäle", ces "salles de paroles" qui s'ouvrent la nuit? Plus loin Binswanger précise qu'il faut avoir à l'esprit le salon d'accueil où le malade retrouve les visiteurs de sa famille, et doit "s'expliquer" avec le passé et subir des jugements; le parloir est une "némésis", le "front d'un champ de bataille", le lieu d'une explication avec sa vie antérieure. La salle à parler est aussi un espace centré, le lieu d'une confrontation publique, une scène de théâtre et de mise en accusation.

Le phénoménologue recherche le sens sous le mot, il tente de retrouver l'expérience vécue. Mais cette expérience vécue exige un effort d'entrée, un exercice de familiarisation analogue à celui des artistes dont il était question plus haut. Il faut "entrer dans", se rendre capable de percevoir ("sich der Sache, dem Erlebnis zuwenden", "sich einleben", "hinein versetztwerden" ${ }^{17}$ ).

Ici, dans le cas des voix et de la salle de paroles, c'est la perception même de l'espace qui est en jeu. La malade entend des voix qui sont à la fois loin et près, une malade explique ainsi qu'elle est contact téléphonique depuis son lit (nous sommes en 1920, il n’y a bien sûr pas de téléphones portables ou cellulaires). Il s'agit 'd'une liaison à distance d'une nature psychologiquement curieuse, dont nous n'avons encore nullement éclairci l'essence phénoménologique". Pourtant le malade sait de quoi il parle, il fait l'expérience d'une certaine forme de présence à la fois proche et lointaine. Binswanger va même jusqu'à écrire que le malade, lui, a pénétré l'essence de cette spatialité étrange:

Avec cette explication, vous êtes placé dans la situation de la liaison téléphonique, une "liaison à distance" psychologiquement curieuse, dont nous n'avons encore nullement éclairci l'essence phénoménologique, alors que les schizophrènes sur le fondement de leurs hallucinations, se sont manifestement frayé davantage un chemin jusqu'à l'essence de ce loin et près, de cette présence qui et à la fois là et pas là. ${ }^{18}$

16 Texte allemand p. 55; trad. française p. 102.

17 Texte allemand p. 57.

18 "Durch diesen Aufschluss werden Sie in die Situation der telephonischen Verbindung versetzt, einer auch psychologisch eigenartigen "Fernverbindung", deren phänomenologisches 
Un point ici mériterait une discussion, bien difficile: comment peut-il prétendre que le malade a pénétré l'essence de la situation ("Schizophrene in das Wesen [...] tiefer eingearbeitert haben")? A d'autres endroits Binswanger, plus tard, précisera que les malades n'ont précisément pas la compréhension de leur propre situation, qu'il leur manque l' "Einsicht", la vue ou la saisie de ce qui leur arrive.

"Je suis dans mon lit en conversation", "un parloir est ouvert". Les paroles du schizophrène font référence à une situation difficile à saisir. Comment un objet ou un autre être humain peut-il être à la fois loin et près? De quelle sorte de distance parle-t-on alors, comment est faite cette spatialité si étrange? Ces interrogations sur la spatialité sont l'amorce des recherches à venir de Binswanger, un thème qu'il reprendra inlassablement tout au long de son œuvre. ${ }^{19}$

\section{Une théorie de la personne}

Binswanger prolonge cette discussion des "voix" du schizophrène en élargissant la perspective: il nous faudrait parvenir à concevoir cette sorte de phénomène dans une globalité. Rappelons le point de départ de toute cette discussion: il nous faut non une appréhension analytique qui décompose l'objet, mais une vue "plus totale". Les traits de conduite doivent pouvoir être saisis ensemble, non comme des phénomènes isolés ("isoliert"), mais sur l'arrière fond d'une personnalité ("auf dem Hintergrund eines Ich, einer Person"), en les prenant alors comme "l'expression d'une personne constituée de telle ou telle manière" ("als Ausdruck oder Kundgabe einer so und so gearteten Person" ${ }^{20}$ ). Ainsi nous pourrions "pour le cas de cette expérience vécue de la salle de paroles, voir une personne devant nous, qui est en relation avec des puissances spirituelles obscures et évolue dans une sphère spirituelle totalement différente de la nôtre".

Comment définir cette sorte de globalité d'une personne, dont les voix ou la bizarrerie des distances serait "l'expression"? Comment définir la "sphère spirituelle" spéciale où la vie du malade se déroule? Le parloir, disent les malades, est un lieu de combat ("Kampffront"), "un point fixe hors des évènements, une position sûre face aux questions de la vie" au milieu du manque de

Wesen wir uns noch keineswegs klargemacht haben, während Schizophrene in das Wesen dieses Nah- und doch Fernseins, dieses Da- und doch Nichtdaseins, sich auf Grund ihrer Halluzinationen offenbar tiefer eingearbeitet haben.", p. 58.

19 Voir les passages de la Fuite des idées à propos de la distance et de l'espace de la fête, voir aussi le remarquable texte Le problème de l'espace en psychopathologie, ainsi que les développements relatifs à la "scène de terreur" au centre de laquelle se placent elles-mêmes Ellen West ou Suzanne Urban.

${ }^{20}$ Texte allemand p. 58. 
sérieux de la vie. Car avant cette traversée qu'est la maladie, avant ce "combat de l'esprit" et la décision ou la victoire, la vie manquait de sérieux et de responsabilité.

Voilà le point d'où peut partir le reste: nous avons devant nous une personne qui a été transformée "éthiquement" ou quant à sa "conception intuitive du monde" ("ethisch oder weltanschaulich veränderte Person"). ${ }^{21}$

C'est l'occasion d'un regret évoqué par Binswanger: "Il nous manque ici une théorie satisfaisante des caractères personnels [eine Personalcharakteristik] en général et plus particulièrement de la personnalité schizophrénique [...] Nousavons besoindeconceptsphénoménologiquesfondamentaux,pour pouvoir saisir l'essence de la personne et la fixer phénoménologiquement." 22

Ici Binswanger évoque brièvement, à propos de cette requête d'une théorie générale des caractères et de la personne, les noms de Pfänder et Jaspers. Mais pour nous dont la lecture est si rétrospective, la lacune dont il est question ici, nous savons qu'elle sera comblée quelques années plus tard par la merveilleuse surprise de Sein und Zeit en 1927. Heidegger est venu offrir à Binswanger les concepts et le cadre philosophique dont il ressentait le manque.

\section{La fuite des idées et les manières d'être au monde}

C'est dans cette continuité qu'il faudrait analyser les textes de la "deuxième période", celle où Binswanger s'inspire très fortement de Sein und Zeit. Les études rassemblées sous le titre Sur la fuite des idées sont l'ouvrage le plus ambitieux dans cette phase de la réflexion de Binswanger. Le livre, qui est un recueil d'études séparées, riche de digressions et d'analyses détaillées, est plutôt une sorte de vaste chantier, qu'il est hors de question de présenter ici systématiquement. Donnons quelques indications brèves sur l'usage de la phénoménologie heideggerienne dans ce texte.

Cette fois Binswanger a si l'on peut dire une "théorie de la personne" grâce à l'analytique existentiale de Heidegger, et il se juge en mesure de discerner un noyau fondamental de la personne, à partir duquel on peut dériver ou comprendre de manière unitaire des symptômes ou des traits de conduite.

21 L'argumentation de ce passage crucial est difficile à cerner exactement. Y a-t-il une relation entre le sérieux et l' "éthique"? Faut-il supposer que le "sérieux" gagné par lutte spirituelle ("Geisterkampf"), le sens de la responsabilité que le malade pense avoir acquis, constituent le coeur d'une personnalité nouvelle et comme le noyau d'où découlent les expressions? Faut-il prendre "ethisch, oder wenn Sie wollen, weltanschaulich verändert" comme un pléonasme, dans lequel "ethisch" aurait un sens assez large et vague, presque équivalent à "weltanschaulich"? Ou bien "ethisch" fait-il référence à ce sens du sérieux et de la décision nouvellement gagné par le malade?

${ }^{22}$ Allemand pp. 58-59. 
La manie, déjà étudiée par Kraepelin, par Abraham, et bien d'autres, lui paraît pouvoir être décrite comme un mode d'être au monde. En particulier la fuite des idées, qui est l'un des symptômes majeurs, peut être interprétée au sein d'une structure existentielle d'ensemble:

pour nous il s'agit de chercher à comprendre l'état de fait de la fuite des idées à partir de la structure d'ensemble de l'homme atteint de fuite des idées. ${ }^{23}$

Par exemple le monde du malade est devenu plus petit, et le malade est capable de sauter d'une chose à l'autre, en négligeant les intermédiaires:

Cela provient du fait que pour la malade tout - pensées, personnes, choses - est davantage rapproché l'un de l'autre "dans l'espace", de sorte qu'elle a "tout sous la main" beaucoup plus près et plus facilement. Son espace vital, son monde sont devenus autres, et c'est seulement à partir de cette modification de son monde qu'il faut comprendre la modification de son comportement, son comportement lui-même. ${ }^{24}$

Si l'on s'en tient à l'espace objectif et mesurable, l'essentiel nous échappe. La question est celle de l'espace vécu, de la distance de "proximité saisissable" des objets pour un individu humain. Proximité des objets, facilité des projets, sentiment de toute puissance, font partie d'une même forme d'existence, d'un style commun et global. Heidegger est ici un auxiliaire précieux, exposant comment la spatialité dérive d'un mode de présence au monde:

Nous appliquons ici les analyses heideggériennes d'ontologie fondamentale pour des finalités anthropologiques-psychiatriques. ${ }^{25}$

De même, l'analyse par Heidegger de la "déchéance" (Verfallen) de la présence humaine est aussi à reprendre sï l'on veut montrer comment le "bond incessant", la "dispersion", l'oubli de soi dans le "tourbillon", sont une forme de temporalité et d'être au monde, c'et à dire un des possibles de l'existence. ${ }^{26}$

Mais Binswanger revendique une distance critique par rapport au maître. Selon Heidegger, l'existence humaine est dominée par le "souci". Cependant, pour comprendre la manie, pour donner tout son sens existentiel à cette forme d'être au monde, il faut pouvoir accepter que l'existence humaine ne se

23 Sur la fuite des idées, trad. par M. Dupuis, Grenoble, Jérôme Millon, 2000, p. 38.

24 Ibidem, p. 40.

25 Ibidem, p. 41, note.

${ }^{26}$ Voir ibidem, pp. 266-267, où Binswanger cite et utilise les $\S \S 35-38$ de Sein und Zeit, comme il le fera à bien d'autres reprises, par exemple dans le Cas Suzanne Urban, p. 86-87 (all. pp. 279-281). 
borne pas au souci, qu'elle peut être aussi non-problématique et allant de soi. ${ }^{27}$ La danse et la fête sont aussi des formes de vie, des modes de présence: dans la danse on ne va pas quelque part, on ne se préoccupe pas d'un but à atteindre, l'espace est structuré plus librement et sans limite. ${ }^{28}$

Dans ce contexte Binswanger rappelle l'importance de la verticalité dynamique, avec la montée et la chute, cette dimension de l'espace vécu qu'il a mise en avant en 1930 dans son court texte Rêve et existence: la spatialité de l'existence inclut le proche et le lointain, le haut et le profond, mais aussi la montée et la chute. ${ }^{29}$

\section{Retour à Husserl ?}

La troisième période des années 1960-1965, celle qui est nommée improprement mais commodément "retour à Husserl", apparaît comme une surprise et un renouveau théorique étonnant. Binswanger sépare désormais les deux formes de théories phénoménologiques qu'il avait traitées comme proches et parfois interchangeables.

\section{a) Le voisinage Husserl-Heidegger}

Quelle est la différence, aux yeux de Binswanger, entre la phénoménologie husserlienne et celle de Heidegger? La question est complexe. Dans les textes des années 1930-1955 Heidegger et Husserl sont souvent traités comme deux doctrines très voisines, avec des nuances et des accents différents. C'est plutôt Heidegger qui est lạ source d'inspiration, notamment par ses analyses sur l'affectivité et la Stimmung, sa description de l'existence inauthentique, sa dérivation de' la spatialité à partir de l'existence, etc. Mais la doctrine de Husserl est parfois mise en parallèle comme si les deux penseurs finalement avançaient des positions très voisines.

Ainsi, lorsque Binswanger se demande, dans la Fuite des idées, quel principe d'ordre on pourrait trouver pour comprendre la fuite "incohérente" des idées, à quelle logique obéit en profondeur ce dérèglement apparemment chaotique de l'expression linguistique, et à quel niveau un ordre est à trouver, loin en amont de l'ordre encore sensé de la fuite "ordonnée" des idées, il met au grand jour ce qu'on pourrait appeler sa stratégie théorique: il faut "déplacer le point de départ en amont", remonter plus haut dans la structure existentielle. Nous avions "compris", dit-il, la fuite ordonnée des idées, comme dérivée d'une certaine structure existentielle, il s'agit maintenant de "comprendre" un désordre plus grand. Est-ce encore possible? Trouverons-nous un "point de

\footnotetext{
27 Ibidem, p. 66.

28 Ibidem, p. 55-56.

${ }^{29}$ Passim, par exemple p. 47 note.
} 
départ" à partir duquel cette fuite grave des idées serait l'expression d'une structure? Il faut, comme chaque fois, recourir à Husserl et à Heidegger:

Toute la différence par rapport à la fuite ordonnée des idées consisterait donc seulement à déplacer le point de départ de l'étude un peu "en arrière", sur la "voie" qui, du sujet transcendantal phénoménologique, conduit à une transcendance constituée (Husserl) ou qui, du Dasein, conduit à son projet de monde, sa transcendance. (Heidegger) ${ }^{30}$

Ici comme dans d'autres textes, le point essentiel et commun aux deux pensées de Husserl et Heidegger, c'est donc que le monde n'est pas donné, le monde est "constitué" ou "projeté", il n'a de réalité que comme le produit ou le corrélat d'une activité intentionnelle ou d'une présence.

Pour caractériser cette "irréalité" du monde, Binswanger cite dans la Fuite des idées une phrase de Husserl, tirée de Logique formelle et logique transcendantale, qui est une sorte de leitmotiv réorchestré dans Manie et Mélancolie et dans Délire: "Le monde réel ne réside que dans la présomption constamment prescrite que l'expérience continuera constamment de se dérouler selon le même style constitutif." ${ }^{31}$ On voit sur cette phrase comment le projet de monde heideggérien peut rejoindre la constitution des transcendances selon Husserl. Peu importe que l'on prenne le point de départ dans un sujet transcendantal ou dans un Dasein.

\section{b) Deux voies distinctes}

Les derniers textes proposent une rupture plus franche entre deux voies: l'herméneutique de Heidegger et la phénoménologie de Husserl. À 80 ans Binswanger prend une orientation nouvelle: non plus décrire le monde du malade, comme une totalité douée de sens avec son style propre, mais exposer l'enchaînement de l'expérience pour repérer ce qui fait défaut ou lacune. La théorie husserlienne de la "constitution" et de la genèse de l'expérience lui sert à décrire et comprendre les "ratages" de l'expérience du réel. Le cadre théorique n'est donc plus celui, heideggérien, de l'herméneutique du Dasein, mais celui, husserlien, de la constitution de l'expérience. On ne décrit plus différents "modèles d'expérience" - ce qui serait de la Daseinsanalyse - on tente de décrire la construction - réussie ou ratée - de l'expérience, avec ses maillons, ses articulations, ses enchaînements. On passe ainsi, dit Binswanger, de la Daseinsstruktur à l'Erfahrungsstruktur, de la structure existentielle à la structure d'expérience. Cela suppose une vue plus génétique et dynamique de l'accès au réel, non plus "comment un malade voit le monde", mais comment vient à se constituer le monde d'un malade (ou d'un homme sain).

30 Sur la fuite des idées, p. 153.

${ }^{31}$ Formale und Transzendantale Logik, 1929 p. 222. In Fuite des idées p. 92 et 286; in Mélancolie et manie, p. 22 et 49 de la trad. française. 
Sur cette différence désormais acquise (en 1960 au moins) entre phénoménologie et Daseinsanalyse, le texte de Délire est assez précis dans son vocabulaire technique:

Par ce terme de description phénoménologique nous voulons exprimer qu'il ne s'agit plus ici du "modèle d'expérience" délirant, ce modèle qui est à comprendre non phénoménologiquement, mais daseinsanalytiquement, c'est à dire à partir de l'herméneutique daseinsanalytique de la maladie qu'est le délire et à partir de l'accomplissement existentiel du malade délirant, [...] il s'agit au contraire de décrire les structures phénoménologiques et les articulations structurelles dans la constitution et la genèse de l' "expérience" délirante. ${ }^{32}$

Pour rendre compte de la défaillance dans l'expérience du malade, il faut d'abord clarifier la manière dont se constitue une expérience perceptive "normale". Ce qui est donné immédiatement, c'est un chaos d'impressions, unifiées par une synthèse; ${ }^{33}$ dans cette synthèse les actes de reprise temporelle, c'est à dire la rétention du passé proche et la protention vers le futur, jouent un rôle décisif. Ce sont principalement ces articulations temporelles qui sont lésées dans l'expérience que fait le malade. Le réel est inséparable des opérations d'enchaînement temporel. ${ }^{34}$ Le "laisser-être" d'un objet suppose, de la part du sujet percevant, un jeu d'attentes et de reprises, que le malade ne peut plus opérer.

Voilà le programme de travail que propose Binswanger au soir de sa vie. Ces directions de recherche nouvelles, assez radicales, sont comme un nouveau départ dans l'usage de la phénoménologie. Pour les approfondir, les reprendre de manière critique et en tirer toute la fécondité, il faudrait pouvoir entrer dans le détail de la théorie de Husserl sur les "synthèses passives", y compris à partir de textes husserliens aujourd'hui publiés que Binswanger n'a pas pu connaître directement.

32 Délire, p. 33 de la trad. française; texte allemand Wahn, 1965, p. 36: "Mit dem Wort (phänomenologische) Beschreibung wollen wir zum Ausdruck bringen, daß es uns hier nicht mehr auf das wahnhafte "Erfahrungsmodell", das gar nicht phänomenologisch, sondern nur daseinsanalytisch, nämlich aus der daseinsanalytischen Hermeneutik der Wahnkrankheit und des Daseinsvollzugs des Wahnkranken zu verstehen ist [...] sondern auf die Beschreibung phänomenologischer Strukturen und Strukturenzusammenhänge in der Konstitution und Genesis der wahnhaften "Erfahrung" ankommt."

33 Wahn, p. 37: "Unmittelbar gegeben ist uns nur ein Chaos von "Eindrücken"."; p. 38: "Anschauungen können wir daher bezeichnen als synthetisch gestaltete Einheiten von Eindrücken."

34 Binswanger reprend sous cet éclairage nouveau la proposition qu'il avait extraite de la Logique de Husserl: "Die Realität überhaupt ist, wie wir wissen, nur möglich unter der beständigen vorgezeichneten Präsumption, daß die Erfahrung beständig im selben Stil fortlaufen werde." (Wahn, p. 55). 


\section{Le cas Suzanne Urban}

Embrassant ce paysage intellectuel dans son ensemble, depuis l'éblouissement de la découverte de l'intuition au sens large, vers 1920, puis avec la merveilleuse surprise de Sein und Zeit, et enfin avec le tournant tardif des synthèses passives, on ne peut qu'admirer l'ampleur et la force de l'œuvre de Binswanger.

Un lecteur qui voudrait entrer dans le mouvement si riche de cette pensée pourrait se décourager ou s'effrayer: par où commencer? que lire d'abord ? À Jacqueline Verdeaux qui venait le voir avec son ami Michel Foucault, il avait suggéré de traduire d'abord Rêve et existence, ce texte si court et si énigmatique de 1930 - le thème en est d'une certaine manière la chute, la perte du sol et de la verticalité. La traduction est parue en 1954, revue par ses soins et préfacée par Foucault. Ce texte si dense et si étonnant peut servir de prélude comme en musique, mais pas d'introduction méthodique. Plus tard Binswanger a demandé à la même traductrice de rendre en français le Cas Suzanne Urban, où il voyait son testament psychiatrique. C'est par là que nous suggérons de commencer, même si les justifications méthodiques n'apparaissent pas toujours explicitement, et si cette étude est en quelque sorte intermédiaire, tout à fait heideggérienne pour l'essentiel, mais avec des aspects nouveaux.

\section{ABSTRACT}

Pour élaborer son analyse existentielle, Ludwig Binswanger a utilisé la phénoménologie. Mais qu'appelait-il exactement ainsi ? Sa définition de la phénoménologie et l'usage qu'il en fait ont varié, depuis la présentation aux psychiatres donnée en 1922 («De la phénoménologie»), en passant par la reprise de Heidegger à l'époque de la «Fuite des idées» (1933), puis les critiques adressées à «Sein und Zeit» et la construction d'une anthropologie dans les «Grundformen» (1942), enfin avec l'ultime et étonnant tournant husserlien de «Mélancolie et Manie» (1960) et «Délire» (1965). 
